
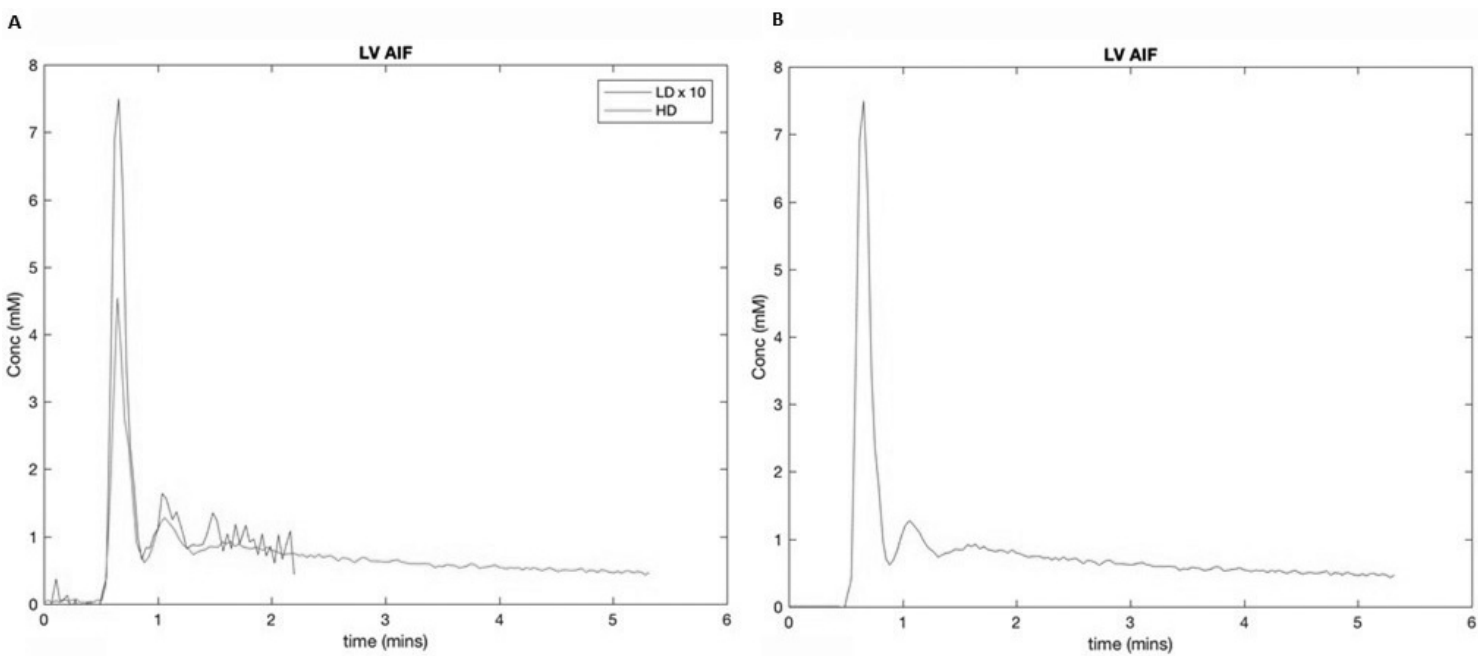

Abstract 17 Figure 1 LV arterial input function (AIF); LD - low dose dotarem (0.005 mmol/kg), HD - high dose dotarem (0.05 mmol/kg) Part A: Before merging of the curves. Part B: After merging of the curves.

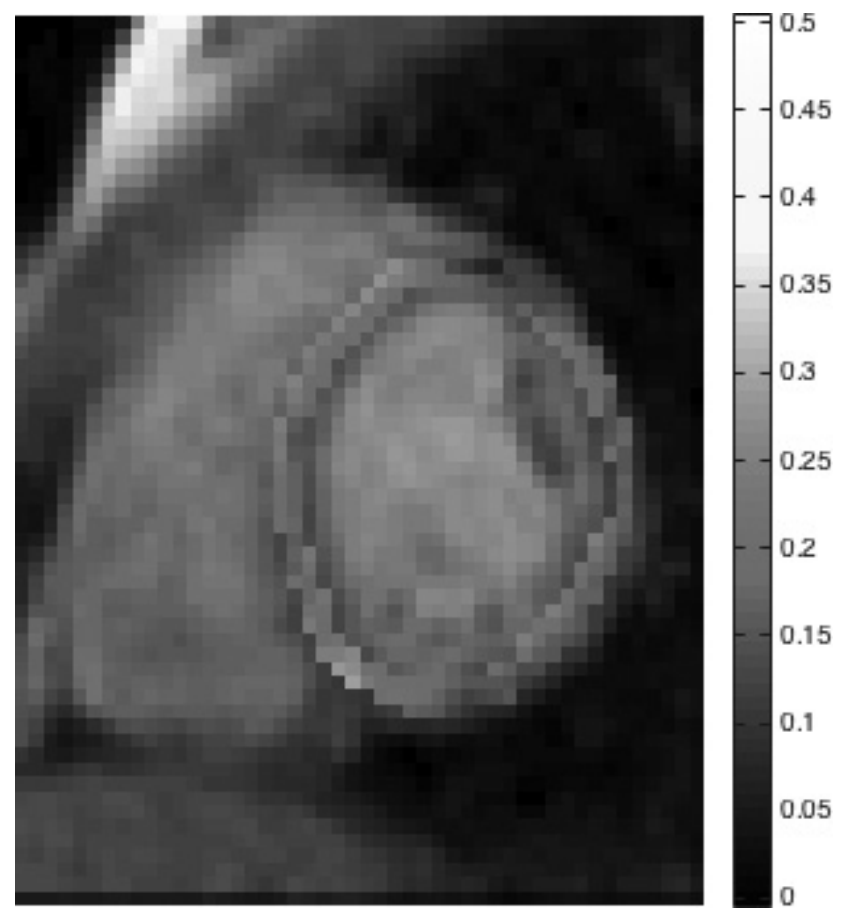

Abstract 017 Figure 2 Myocardial extracellular volume map.

Results Parametric mapping and LGE imaging provides direct tissue characterisation.

Myocardial and lung contrast agent kinetics are modelled from the DCE images in matlab (Extended Kety Model) to determine heart and lung capillary permeability (Ktrans), extracellular volume (ECV) and plasma volume fraction (vp). Regions of interest are drawn in the LV and RV cavities to obtain arterial input functions (AIF). The low dose AIF is multiplied by a factor of ten and the first pass peak is combined with the high dose AIF curve in order to correct for saturation effects (Figure 1).

Examples of ECV maps are shown in Figure 2 and 3.

Conclusions This combined heart lung protocol is an efficient and comprehensive imaging tool for simultaneous myocardial

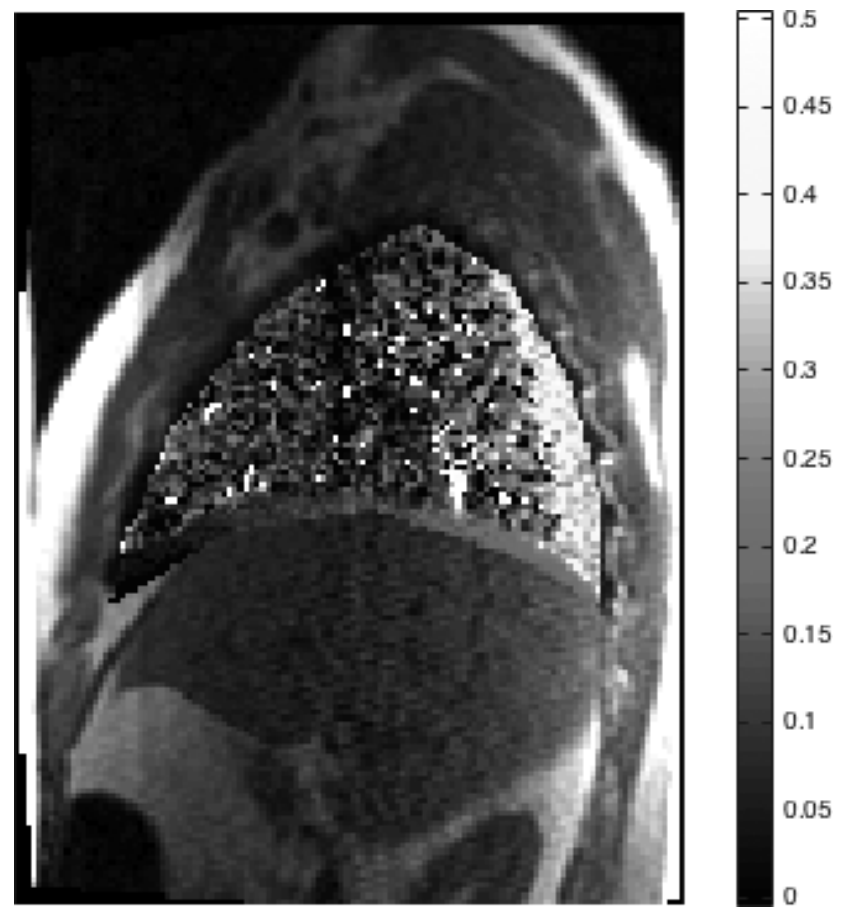

Abstract 017 Figure 3 Lung extracellular volume map.

and pulmonary assessment. It has a potential to help elucidate complex links between pulmonary and myocardial pathologies, and is a model for other dual organ parametric MR imaging.

\section{DT-CMR IMAGING OF DERANGED MICROSTRUCTURE IN SITUS INVERSUS TOTALIS}

${ }^{1} Z$ Khalique, ${ }^{1}$ PF Ferreira, ${ }^{1} \mathrm{AD}$ Scott, ${ }^{1,2} \mathrm{~S}$ Nielles-Vallespin, ${ }^{1} \mathrm{DN}$ Firmin, ${ }^{1} \mathrm{DJ}$ Pennell. ${ }^{1} \mathrm{NIHR}$ CBRU, Royal Brompton Hospital, London, UK; ${ }^{2} \mathrm{NIH}$, Bethesda, USA

10.1136/heartjn|-2017-311399.18

Background Diffusion tensor cardiovascular magnetic resonance (DT-CMR) is a non-invasive technique that has been 
histologically validated to provide information on the microstructure of the myocardium. ${ }^{1-3}$ There is a standard helical myocyte arrangement in the situs solitus (SS) heart, as viewed from the apex; left-handed in the epicardium, circumferential in the mesocardium and right-handed in the endocardium. This generates the opposing basal clockwise rotation and apical anticlockwise rotation necessary for torsion. ${ }^{4}$

However in situs inversus totalis (SIT) there is mirror image arrangement of the visceral organs. There are no histological studies assessing how myocardial microstructure is affected in SIT. DT-CMR offers a unique opportunity to evaluate the microstructural abnormalities in SIT.

Methods 12 SIT patients and 12 matched healthy volunteers were scanned in a $3 \mathrm{~T}$ Siemens Skyra scanner. DT-CMR was performed at peak systole in the short axis at base, mid and apex. 2 people had whole heart tractography. A STEAM sequence with bmain $=600 \mathrm{~s} / \mathrm{mm}^{2}$ and bref $=150 \mathrm{~s} / \mathrm{mm}^{2}$ was used, as previously described. ${ }^{3}$ Strain and torsion assessment was performed using DENSE5.

Results Patients were age and sex-matched (SIT 39.5 and SS 34.5 years, 3/12 male). Myocyte organisation at base, mid and apex was significantly different in SIT hearts compared to the SS hearts. Figure 1 shows example tractography. In SS, the endocardial positive helix angle (HA) progressed to a negative HA in the epicardium. In SIT hearts this pattern was inverted at the base and approached normal at the apex, with a midventricular transition zone.

Mid-ventricular peak radial and circumferential strain were reduced in SIT $(0.4 \pm 0.16$ vs $0.56 \pm 0.16, p=0.02$, and -0.16 \pm 0.02 vs $-0.18 \pm 0.01 \mathrm{p}=0.04$ respectively). Peak absolute torsion was reduced in SIT $3.6^{\circ}\left[3.6^{\circ}\right]$ vs $8.0^{\circ}\left[3.5^{\circ}\right]$.

Conclusion This is the first human DT-CMR study of SIT hearts. We demonstrate that the SIT heart has an inverted myocyte orientation at the base that approaches normal towards the apex. Peak absolute torsion is reduced. Peak radial and circumferential strain are also reduced at the midventricle. The deranged microstructure in SIT has functional effects, the long-term outcomes of which require further study.

\section{REFERENCES}

1. Reese TG, Weisskoff RM, Smith RN, et al. Imaging myocardial fibre architecture in vivo with magnetic resonance. Magn Reson Med 1995;34(6):786-791.

2. Scollan DF, Holmes A, Winslow $R$, et al. Histological validation of myocardial microstructure obtained from diffusion tensor magnetic resonance imaging. $\mathrm{Am} J$ Physiol Heart Circ Physiol 1998;275(6):H2308-H2318.

3. Nielles-Vallespin S, Khalique Z, Ferreira PF, et al. Assessment of Myocardial Microstructural Dynamics by In Vivo Diffusion Tensor Cardiac Magnetic Resonance. J Am Coll Cardiol 2017;69(6):661-676.

4. Sengupta PP, AJ Tajik, K Chandrasekaran, et al. Twist Mechanics of the Left Ventricle: Principles and Application. JACC: Cardiovascular Imaging 2008;1(3):366376.

5. Scott AD, Tayal U, Nielles-Vallespin $S$, et al. Accelerating cine DENSE using a zonal excitation. J Cardiov Magn Reson 2016;18(1):050.

\section{CHEMOTHERAPY-INDUCED CARDIOTOXICITY: COULD A TRANSLATIONAL CARDIAC MRI MODEL HELP IDENTIFY PATIENTS AT RISK?}

${ }^{1,2} \mathrm{~L}$ Cove-Smith*, ${ }^{3} \mathrm{M}$ Schmitt, ${ }^{1} \mathrm{C}$ Dive, ${ }^{1} \mathrm{~A}$ Backen, ${ }^{2} \mathrm{~N}$ Mescallado, ${ }^{4} \mathrm{R}$ Roberts, ${ }^{4} \mathrm{H}$ Mellor, ${ }^{5} \mathrm{D}$ Morris, ${ }^{5}$ J Naish, ${ }^{5} \mathrm{~A}$ Jackson, ${ }^{4} \mathrm{~J}$ Kirk, ${ }^{4} \mathrm{~A}$ Hargreaves, ${ }^{4} \mathrm{M}$ Galvin, ${ }^{4} \mathrm{~S}$ Smith, ${ }^{4} \mathrm{~S}$ Brocklehurst, ${ }^{4} \mathrm{~S}$ Price, ${ }^{4} \mathrm{C}$ Betts, ${ }^{4} \mathrm{P}$ Hockings, ${ }^{4} \mathrm{~N}$ Woodhouse, ${ }^{2,6} \mathrm{~J}$ Radford, ${ }^{2,6} \mathrm{~K}$ Linton. ${ }^{1}$ Clinical and Experimental Pharmacology, The Cancer Research UK Manchester Institute, University of Manchester, Manchester, UK; ${ }^{2}$ Medical Oncology, The Christie NHS Foundation Trust, Manchester, UK; ${ }^{3}$ Cardiology, University Hospital of South Manchester, Manchester, UK; ${ }^{4}$ Astrazeneca, Macclesfield, UK; ${ }^{5}$ Biomedical Imaging Institute, University of Manchester, Manchester, UK; ${ }^{6}$ Institute of Cancer Sciences, University of Manchester, Manchester, UK

\subsection{6/heartjnl-2017-311399.19}

Background Cancer survivorship is an international priority and mortality from cardiac damage is one of the greatest concerns. Anthracycline chemotherapy is cardiotoxic but remains highly effective against cancer.

Methods This translational project involved the development of a pre-clinical rat model of progressive anthracycline-induced cardiotoxicity $\left(1.25 \mathrm{mg} / \mathrm{m}^{2}\right.$ doxorubicin weekly for 8 doses $)$ and a concurrent clinical study of 30 cancer patients receiving 6 cycles of curative anthracycline therapy (doxorubicin $50 \mathrm{mg}$ / $\mathrm{m}^{2}$ ). A bespoke cardiac magnetic resonance imaging (CMR) protocol was developed which included longitudinal T1

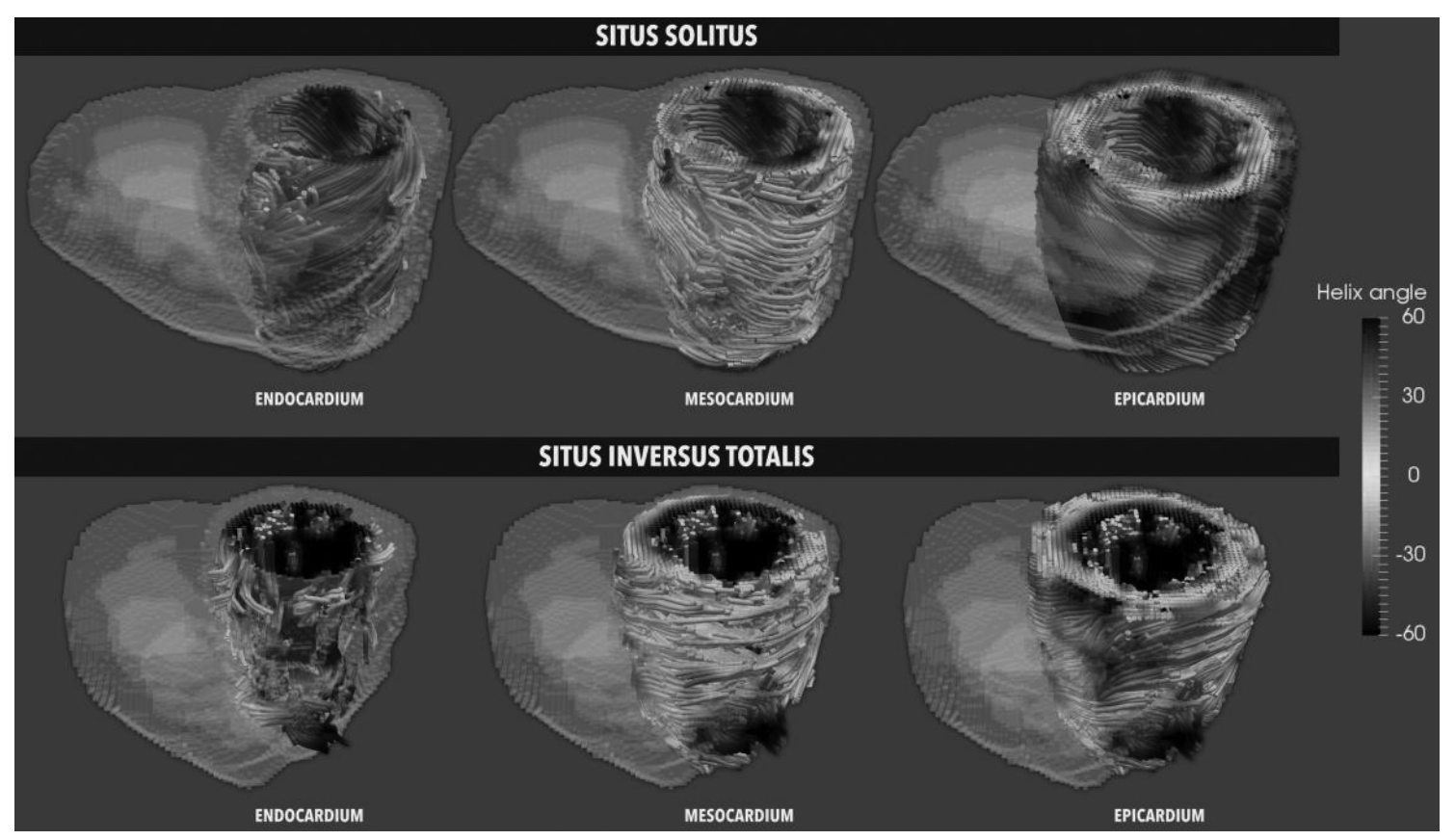

Abstract 18 Figure 1 Tractography of a SIT and SS heart. 